\title{
Treatment Failure and Costs in Patients With Methicillin-Resistant Staphylococcus aureus (MRSA) Skin and Soft Tissue Infections: A South Texas Ambulatory Research Network (STARNet) Study
}

\author{
Matthew J. Labreche, PharmD, MS, Grace C. Lee, PharmD, \\ Russell T. Attridge, PharmD, MS, Eric M. Mortensen, MD, MS, Jim Koeller, MS, \\ Liem C. Du, MD, Natalie R. Nyren, PA-C, Lucina B. Treviño, MD, \\ Sylvia B. Treviño, PA-C, Joel Peña, MD, Michael W. Mann, MD, Abilio Muñoz, MD, \\ Yolanda Marcos, MD, Guillermo Rocha, MD, Stella Koretsky, MD, \\ Sandra Esparza, MD, Mitchell Finnie, MD, Steven D. Dallas, PhD, \\ Michael L. Parchman, MD, PhD, and Christopher R. Frei, PharmD, MS
}

Objective: To measure the incidence of treatment failure and associated costs in patients with methicillin-resistant Staphylococcus aureus skin and soft tissue infections (SSTIs).

Methods: This was a prospective, observational study in 13 primary care clinics. Primary care providers collected clinical data, wound swabs, and 90-day follow-up information. Patients were considered to have "moderate or complicated" SSTIs if they had a lesion $\geq 5 \mathrm{~cm}$ in diameter or diabetes mellitus. Treatment failure was evaluated within 90 days of the initial visit. Cost estimates were obtained from federal sources.

Results: Overall, treatment failure occurred in $21 \%$ of patients $(21$ of 98$)$ at a mean additional cost of \$1,933.71 per patient. In a subgroup analysis of patients who received incision and drainage, those with moderate or complicated SSTIs had higher rates of treatment failure than those with mild or uncomplicated SSTIs (36\% vs. $10 \% ; P=.04)$.

Conclusions: One in 5 patients presenting to a primary care clinic for a methicillin-resistant $S$. aureus SSTI will likely require additional interventions at an associated cost of almost $\$ 2,000$ per patient. Baseline risk stratification and new treatment approaches are needed to reduce treatment failures and costs in the primary care setting. (J Am Board Fam Med 2013;26:508-517.)

Keywords: Antibiotics, Cost of Illness, Epidemiology, Infectious Diseases, Practice-based Research, Primary Health Care

Community-acquired (CA) methicillin-resistant Staphylococcus aureus (MRSA) skin and soft tissue

This article was externally peer reviewed.

Submitted 4 September 2012; revised 20 December 2012; accepted 2 January 2013.

From the Division of Pharmacotherapy, College of Pharmacy, University of Texas at Austin, Austin (MJL, GCL, JK, $\mathrm{CRF}$ ); the Pharmacotherapy Education and Research Center, School of Medicine (MJL, GCL, JK, CRF); the South Texas Ambulatory Research Network (LCD, NRN, LBT, SBT, JP, MWM, AM, YM, GR, SK, SE, MF); the School of Health Professions, Clinical Laboratory Sciences (SDD), University of Texas Health Science Center, San Antonio; the Feik School of Pharmacy, University of the Incarnate Word, San Antonio, TX (RTA); the Division of General Internal Medicine, VA North Texas Health Care System, Dallas (EMM); the Departments of Internal Medicine and Clinical Sciences, University of Texas Southwestern Medi- infections (SSTIs) are commonplace in community health care settings. ${ }^{1-4}$ Furthermore, patients with CA-MRSA SSTIs have a high incidence of treat-

cal Center, Dallas (EMM); and the MacColl Center for Health Care Innovation, Group Health Research Institute, Group Health Cooperative, Seattle, WA (MLP).

Funding: This study was funded by an investigator-initiated research grant from Pfizer (CRF). Support also was provided by the U.S. National Institutes of Health in the form of a KL2 Career Development Award (NIH/NCRR 5KL2 RR025766) (CRF).

Conflict of interest: none declared.

Corresponding author: Christopher R. Frei, PharmD, MS, Pharmacotherapy Education and Research Center, School of Medicine, University of Texas Health Science Center at San Antonio, 7703 Floyd Curl Dr., MC 6220, San Antonio, TX 78229-3900 (E-mail: freic@uthscsa.edu). 
ment failure and recurrence. Two recent studies involving urgent and primary care clinics found that $35 \%$ of patients with CA-MRSA SSTIs experienced treatment failure and $78 \%$ of patients reported a recurrent infection. ${ }^{5,6}$ Reasons for treatment failure may be multifactorial and include inappropriate antibiotics, antibiotic resistance, and disease severity; however, studies evaluating mechanisms of treatment failure are limited.

The Infectious Diseases Society of America (IDSA) has published 2 evidence-based practice guidelines related to the care of patients with MRSA SSTIs. ${ }^{7,8}$ The 2005 SSTI guidelines recommend incision and drainage (I\&D) for purulent SSTIs. ${ }^{7}$ I\&D is an important treatment modality for MRSA SSTIs because approximately $80 \%$ to $90 \%$ will present as purulent infections. ${ }^{4,9}$ Systemic antibiotics are recommended for those patients with multiple lesions, impaired host defenses, extensive cellulitis surrounding the infection, cutaneous gangrene, and systemic manifestations. ${ }^{7}$

The 2011 MRSA guidelines also emphasize the use of I\&D for MRSA SSTIs with drainable foci. ${ }^{8}$ These guidelines recommend adjunctive antibiotics for the same populations identified in the 2005 IDSA SSTI guidelines as well as patients at extremes of age, those with SSTIs in areas that are difficult to drain (eg, face, hand, genitalia), and in those who do not respond to I\&D alone. The 2011 IDSA MRSA guidelines define the "impaired host defenses" category as those patients with diabetes, neoplasms, and human immunodeficiency virus (HIV)/ acquired immunodeficiency syndrome (AIDS). The guidelines reiterate that these patients should receive adjunctive antibiotics regardless of other patient characteristics. These recommendations are supported by a recent systematic review that suggests oral generic antiMRSA antibiotics (eg, clindamycin, doxycycline, and trimethoprim-sulfamethoxazole) may provide additional benefit to I\&D alone for purulent MRSA SSTIs. ${ }^{10}$ Taken together, this information indicates that I\&D plus anti-MRSA antibiotics is the standard of care for most patients with purulent MRSA SSTIs.

There are several severity classification rules for SSTIs $^{7,8,11-13}$; however, the best classification rule is unknown. ${ }^{14}$ Without a well-recognized rule, patients with large abscesses or significant comorbidities, including diabetes, may receive less aggressive therapy than is warranted. This may be partially responsible for the high rate of treatment failure and recurrence in the community setting.

Several studies have quantified the prevalence of CAMRSA SSTIs in the primary care setting and described the management of these infections. ${ }^{6,15,16}$ Levy and Daly ${ }^{15}$ conducted a large prospective study assessing the management of SSTIs in primary care clinics within the Iowa Research Network. Among their many important findings, the investigators found that patients who received initial I\&D had a shorter time to disease resolution (11.4 days versus 13.6 days) than patients who did not receive initial I\&D. ${ }^{15}$ Members of our own research group have conducted 2 studies investigating CA-MRSA SSTIs involving the South Texas Ambulatory Research Network (STARNet), a practice-based research network. ${ }^{6,16}$ The first study used a "card system" to collect data on patients with CA-MRSA SSTIs seen between April 2007 and January 2008. ${ }^{6}$ The chief findings were identification of CA-MRSA risk factors and high prevalence of CA-MRSA SSTIs (67\%) in this region. The second study enrolled a new set of patients seen in STARNet clinics between 2009 and 2010. ${ }^{16}$ This largely descriptive study involved the collection of clinical data, microbiologic specimens, and digital pictures of the infections. The major findings were the prevalence of CA-MRSA SSTIs (61\%) and a description of the initial treatment patterns in the primary care setting.

The study described in this article is a continuation of the second STARNet CA-MRSA SSTI study. New support enabled us to expand the original cohort, collect 90-day treatment outcomes, and perform a cost assessment. This study investigates possible causes for treatment failure, including failure to incise and drain purulent infections and a reluctance to administer adjunctive antibiotics to patients with more severe infections. Finally, it also provides estimates for the incidence of treatment failure and associated costs.

\section{Methods}

This was a multisite, prospective, communitybased, observational cohort study pursued in collaboration with STARNet and comprising 108 urban, suburban, and rural primary care clinics distributed throughout the south Texas region. Health care providers at 13 STARNet clinics prospectively enrolled patients for this study. Similar to the other STARNet clinics, these 13 clinics serve 
a predominately Hispanic and non-Hispanic white population. Unlike the other STARNet clinics, these 13 clinics provide care to a largely underserved population, many of whom do not have medical insurance. Patients were eligible for enrollment in the study if they provided informed consent, were $\geq 18$ years old, presented with a SSTI to one of the participating clinics, and their managing clinician suspected MRSA. This analysis was further limited to patients confirmed to have MRSA via microbiology tests conducted in the principal investigator's (CRF) research laboratory, those with baseline SSTI severity and treatment data, and those with 90-day follow-up data. No protocols or educational interventions were implemented as part of this observational study. Patients were excluded if they were pregnant, incarcerated, or had impaired decisionmaking capacity. The University of Texas Health Science Center at San Antonio Institutional Review Board granted approval for this study.

Study investigators provided the clinics with English and Spanish informed consent forms, a digital camera, a portable biohazard container, and individually labeled study kits, as previously described. ${ }^{16}$ STARNet clinicians were instructed to obtain informed consent from patients, record demographic and clinical information on the patient data card, obtain a wound culture, crush the ampule to release modified Stuart's media onto the tip of the swab for preservation during transport, and capture digital pictures of the infection site. Clinic staff contacted the principal investigator (CRF) when they enrolled a patient. The principal investigator retrieved the data cards, wound swabs, and pictures from the study sites and returned these materials to his research laboratory for processing. Clinics were compensated $\$ 50$ for each patient enrolled in the study.

Wound swabs were submitted in transport media and then were plated directly onto prefilled tryptic soy agar plates with $5 \%$ sheep blood (Remel, Lenexa, KS) and incubated for 18 to 24 hours at 35 to $37^{\circ} \mathrm{C}$. If the isolate did not grow, the culture was replated up to 2 more times. If growth did not occur after three attempts, "no growth" was recorded. If growth occurred, isolates morphologically consistent with $S$. aureus were subcultured once onto another blood agar plate under the same conditions to facilitate logarithmic growth. Next, the suspect isolates were plated onto MRSASelect chromogenic agar (Bio-Rad Laboratories, Hercu- les, CA) plates for identification and isolation of MRSA; incubation occurred for 18 to 28 hours at 35 to $37^{\circ} \mathrm{C}$, protected from light. Pink colonies on the MRSASelect agar were indicative of MRSA. These methods have been used previously by this research group to identify MRSA SSTIs in a primary care setting. ${ }^{16}$

\section{Classification of Severity}

The severity of the infection was categorized using a modified version of 2 U.S. Food and Drug Administration (FDA) classification schemes. This modified classification scheme has also been used in a prior study. ${ }^{16}$ The FDA's 1998 Guidance for Industry SSTI document defines "complicated" infections as those that penetrate the deeper soft tissues, those for which significant surgical intervention is necessary, and those occurring in patients with comorbid conditions that may hinder treatment response (eg, diabetes mellitus or HIV). ${ }^{12}$ A more recent FDA guidance document classifies abscesses affecting a body surface area $\geq 75 \mathrm{~cm}^{2}$ as "major abscesses" and those affecting an area of $<5 \mathrm{~cm}$ from the peripheral margin of the abscess as "minor abscesses." "13 The modified scheme classifies patients as "moderate or complicated" if they had a lesion $\geq 5 \mathrm{~cm}$ in diameter, diabetes mellitus, or both. Patients who do not exhibit these characteristics are considered to have "mild or uncomplicated" infections.

\section{Health Outcomes}

Participating clinics were asked to collect, via medical chart review, additional follow-up information regarding events that transpired after the initial clinic visit. Clinic personnel used a standardized follow-up form that requested information regarding patient age; medical history (eg, peripheral vascular disease, chronic noninfectious skin disorder, HIV/AIDS, cancer, actively receiving chemotherapy, immunosuppressed at time of visit); type of infection (eg, furuncle, abscess, cellulitis, other); and events occurring within 90 days of the initial clinic visit. This form was collected by the principal investigator (CRF) or returned electronically, void of any protected health information.

Information from the 90-day follow-up was used to assess the incidence of treatment failure. Patients were considered to have experienced treatment failure if any of the following occurred within 90 days of their initial visit: (1) change in antibiotic therapy, 
Table 1. Estimated Additional Costs of Treatment Failure

\begin{tabular}{ll}
\hline Intervention & Cost (2011 US\$)* \\
\hline Hospital admission $^{17}$ & $17,591.07$ \\
Outpatient incision and drainage $^{20}$ & $2,130.96$ \\
Emergency department visit $^{18}$ & 754.84 \\
Antibiotics $^{34}$ & \\
Bacitracin ointment & $5.23 /$ tube \\
Mupirocin ointment & $0.31 / 25 \mathrm{~g}$ \\
Cephalexin 500-mg capsules & $0.11 /$ capsule \\
Ciprofloxacin 500-mg tablets & $0.15 /$ tablet \\
Clindamycin 300-mg capsules & $0.20 /$ capsule \\
Clindamycin 150-mg capsules & $0.07 /$ capsule \\
Doxycycline 100-mg capsules & $0.04 /$ capsule \\
Trimethoprim-sulfamethoxazole & $0.04 /$ tablet \\
$\quad$ double-strength tablets & \\
Clindamycin 600 mg intravenous & $2.24 / 600 \mathrm{mg}$ \\
$\quad$ solution & \\
\hline
\end{tabular}

*All costs were discounted to 2011 U.S. dollars using National Medical Consumer Price Index inflation rates. ${ }^{21}$

(2) I\&D (after initial I\&D or receipt of initial antibiotics only) (3) SSTI at a new site, (4) SSTI at the same site, (5) emergency department visit, or (6) hospital admission.

\section{Data and Statistical Analysis}

JMP 9.0 statistical software (SAS Institute, Cary, NC) was used for all statistical analyses. Fisher exact and $\chi^{2}$ tests were used to compare nominal variables, Student $t$ test was used to compare normally distributed numeric variables, and the Wilcoxon Rank-Sum test was used to compare ordinal and nonnormally distributed numeric variables.

A cost analysis was performed from the perspective of the health insurance payer. As such, indirect medical costs, such as loss of productivity, were not assessed in this study. Direct medical costs (eg, hospital admission and I\&D) were derived using estimates from the Agency for Healthcare Research and Quality. ${ }^{17-20}$ Agency for Healthcare Research and Quality national average drug acquisition costs, obtained from the Centers for Medicare \& Medicaid Services, were used to estimate drug costs (Table 1). In patients with multiple interventions or repeated interventions of the same type during the 90-day follow-up period, the cost per intervention was multiplied by the total number of each type of intervention. All costs were then summed for patients with multiple types of failure (eg, subsequent
I\&D and change in antibiotic therapy). All costs were incurred during a 32-month period (February 2009 to October 2011) and therefore were adjusted to 2011 United States (U.S.) dollars using national medical consumer price index (MCPI) inflation rates. $^{21}$ The following equation was used to perform the cost discounting:

2011 Cost $=$ (cost estimate for resource in year $\mathrm{X}) \times(1+\mathrm{MCPI}$ rate for years between year $\mathrm{X}$ and 2011).

\section{Results \\ Primary Analysis}

A total of 265 cases were collected from 13 primary care clinics over a 32-month period. From 12 clinics, 137 cases had MRSA-positive cultures (MRSA rate, $52 \%$ ). Initial treatment data were missing for 7 patients and data necessary to classify SSTI severity were missing for 5 patients. Of the 125 remaining cases, 98 patients from 10 clinics had 90 day follow-up information available for this analysis. The patients had a mean age of 42 years and most were Hispanic (74\%). More than half of the patients $(52 \%)$ had a body mass index $\geq 30$ $\mathrm{kg} / \mathrm{m}^{2}$, indicating obesity. Many patients had no medical insurance (43\%).

Figure 1 depicts the distribution of patients into the 2 categories of SSTI severity. Nearly twothirds of patients had moderate or complicated MRSA SSTIs. The most common characteristic placing patients into the moderate or complicated severity category was lesion size $\geq 5 \mathrm{~cm}$ in diameter (57\%); $18 \%$ of patients had diabetes alone and $25 \%$ had both characteristics. Patients with moderate or complicated MRSA SSTIs were more likely to be Hispanic $(83 \%$ vs $60 \% ; P=.05)$ and have a body

Figure 1. Severity classification and qualifying characteristics of methicillin-resistant Staphylococcus aureus skin and soft tissue infections $(\mathbf{n}=98)$.

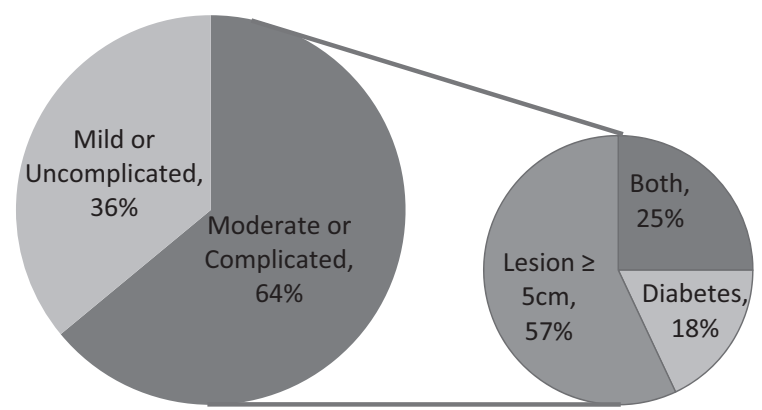


mass index $\geq 30 \mathrm{~kg} / \mathrm{m}^{2}$ (61\% vs $\left.33 \% ; P=.01\right)$ compared with those with mild or uncomplicated MRSA SSTIs. No other characteristics differed significantly between groups.

Of all patients with an MRSA SSTI, 21\% experienced treatment failure ( $\mathrm{n}=21$ of 98 patients). Treatment failure occurred in $27 \%$ of patients in the moderate or complicated group and $11 \%$ of patients in the mild or uncomplicated group $(P=.08)$. In the moderate/complicated group, the failure rate was $60 \%$ for those patients who received I\&D alone and $32 \%$ for those patients who received I\&D plus antibiotics $(P=.33)$. This trend was not present in the mild/uncomplicated group. The mean additional cost of treatment failure was $\$ 1,933.70$ : $\$ 2,093.40$ in the moderate or complicated group versus $\$ 1,255.02$ in the mild or uncomplicated group.

Change in antibiotics was the most common indicator of treatment failure (81\%). A new SSTI was present in $43 \%$ of patients, $24 \%$ required additional I\&D, $24 \%$ were subsequently seen in the emergency department, $10 \%$ had a recurrent SSTI, and $5 \%$ were admitted to the hospital. There were no significant differences in the types of treatment failure between the 2 severity groups $(P>.05$ for all comparisons) (Figure 2).
The mean time to treatment failure was 16.9 days. Time to treatment failure was 11.8 days in the moderate or complicated severity group and 38.8 days in the mild or uncomplicated group $(P=.06)$. More than half (59\%) of all failures in the moderate or complicated severity group occurred within 10 days of the initial clinic visit. In contrast, only $25 \%$ of failures in the mild or uncomplicated occurred within 10 days of the initial clinic visit.

The trunk and lower extremity were the most common infection sites. SSTIs in areas that are difficult to drain (eg, face, hand, genital area) accounted for $13 \%$ of all SSTIs. SSTI location (eg, axilla, buttock, face, groin, hand, head/neck, lower/upper extremities) did not differ significantly between the 2 severity groups.

The most common initial treatments were I\&D plus antibiotics (57\%), antibiotics alone (33\%), and I\&D alone (6\%). Two patients did not receive I\&D or antibiotics at the initial visit; instead they were instructed to return for follow-up at a later date. One patient was referred for surgery. There were no significant differences in initial treatment modality between the 2 severity groups (Table 2).

Overall, 89 of 98 patients (91\%) received antibiotic therapy. The rate of antibiotic therapy was

Figure 2. Type of treatment failure.*

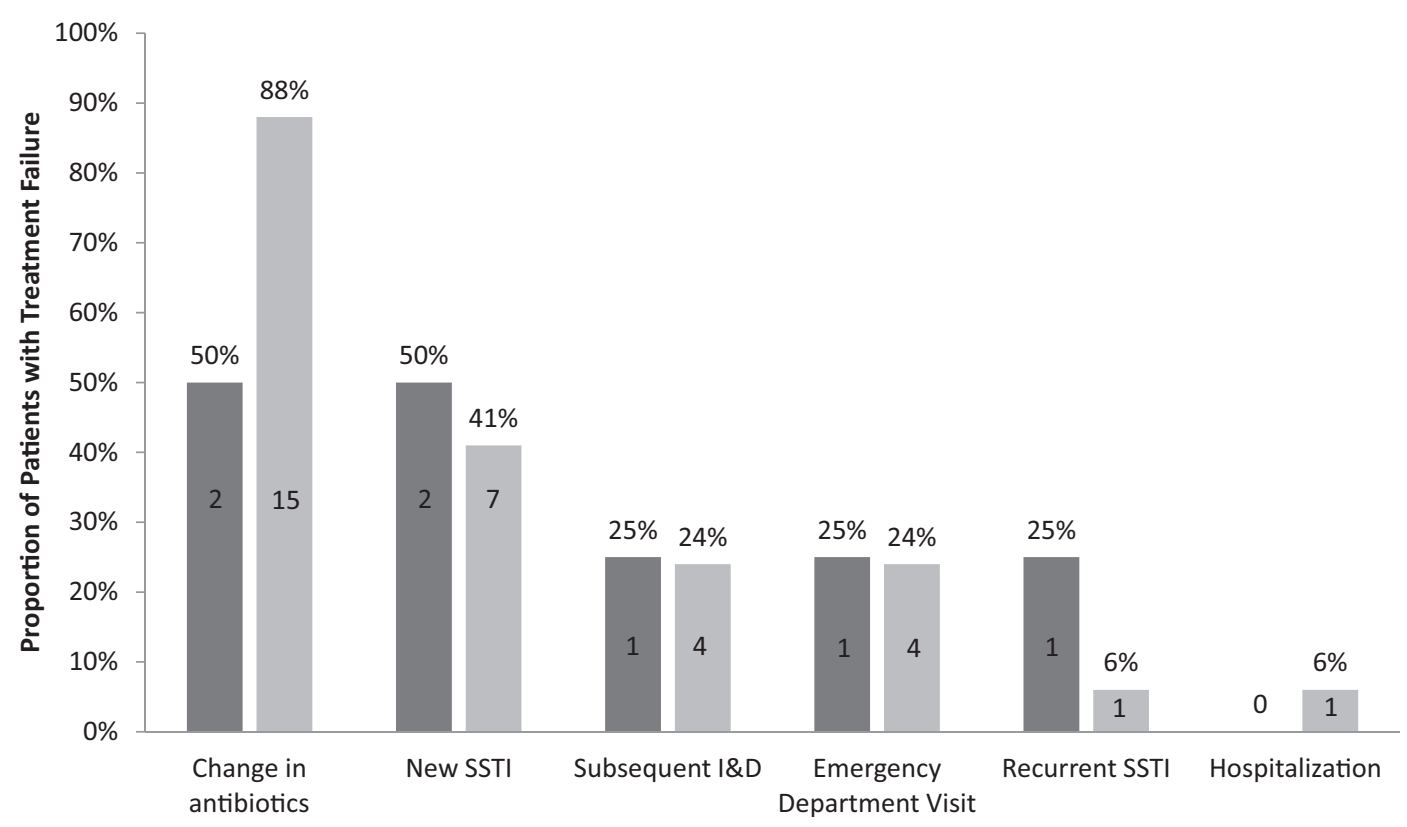

Mild or Uncomplicated, $n=4$ failures in 35 patients Moderate or Complicated, $n=17$ failures in 63 patients

${ }^{*}$ There were no significant differences in the types of treatment failure between the two severity groups $(p>0.05$ for all comparisons). I\&D, incision and drainage; SSTI, skin and soft tissue infections. 
Table 2. Initial Treatment Strategy $(n=98) *$

\begin{tabular}{lccc}
\hline $\begin{array}{l}\text { Treatment } \\
\text { Regimen }\end{array}$ & $\begin{array}{c}\text { Total } \\
(\mathrm{n}=98)\end{array}$ & $\begin{array}{c}\text { Mild or } \\
\text { Uncomplicated } \\
(\mathrm{n}=35)\end{array}$ & $\begin{array}{c}\text { Moderate or } \\
\text { Complicated } \\
(\mathrm{n}=63)\end{array}$ \\
\hline I\&D alone & $6(6)$ & $1(3)$ & $5(8)$ \\
I\&D + antibiotics & $56(57)$ & $19(54)$ & $37(58)$ \\
Antibiotics alone & $33(34)$ & $14(40)$ & $19(30)$ \\
Follow-up alone & $2(2)$ & $1(3)$ & $1(2)$ \\
Surgery & $1(1)$ & $0(0)$ & $1(2)$ \\
\hline
\end{tabular}

Data are shown as $\mathrm{n}(\%)$.

*There was no statistically significant difference in the initial treatment strategy between patients with mild or uncomplicated infections compared with patients with moderate or complicated infections $\left(P=.66, \chi^{2}\right.$ test for homogeneity).

$\mathrm{I} \& \mathrm{D}$, incision and drainage.

similar between the mild or uncomplicated and moderate or complicated severity groups $(94 \%$ vs $88 \%)$. Of those receiving antibiotic therapy, the most common antibiotic regimens were trimethoprim/sulfamethoxazole monotherapy (72\%), combination therapy (18\%), doxycycline monotherapy (3\%), clindamycin monotherapy (3\%), cephalexin monotherapy (2\%), and mupirocin ointment monotherapy (1\%). Of all the combination therapy regimens, $75 \%$ contained trimethoprimsulfamethoxazole. Figure 3 illustrates the initial choice of antibiotic for the 2 severity groups. There were no significant differences in the use of antibiotics or the selection of initial antibiotic therapies between the 2 severity groups.

\section{Subgroup Analysis: Patients Receiving IED}

When the analysis was limited to only those patients who received $I \& D$, the most common initial treatment was I\&D plus antibiotics $(90 \%) ; \mathrm{I} \& \mathrm{D}$ alone was performed on the remaining $10 \%$ of patients. Once again, there were no significant differences in initial treatments between the 2 severity groups.

Of the patients with an MRSA SSTI who received I\&D, 27\% experienced treatment failure ( $\mathrm{n}=17$ of 62 patients). Moderate or complicated patients were more than 3 times as likely to experience treatment failure compared with mild or uncomplicated patients (36\% vs $10 \% ; P=.04$ ) (Figure 4). There were no significant differences in the type of treatment failure between the 2 severity groups $(P>.05$ for all comparisons). The overall mean time to treatment failure was 16.6 days. The mean time to treatment failure was 12.3

Figure 3. Initial antibiotic choice $(\mathrm{n}=98)$.*

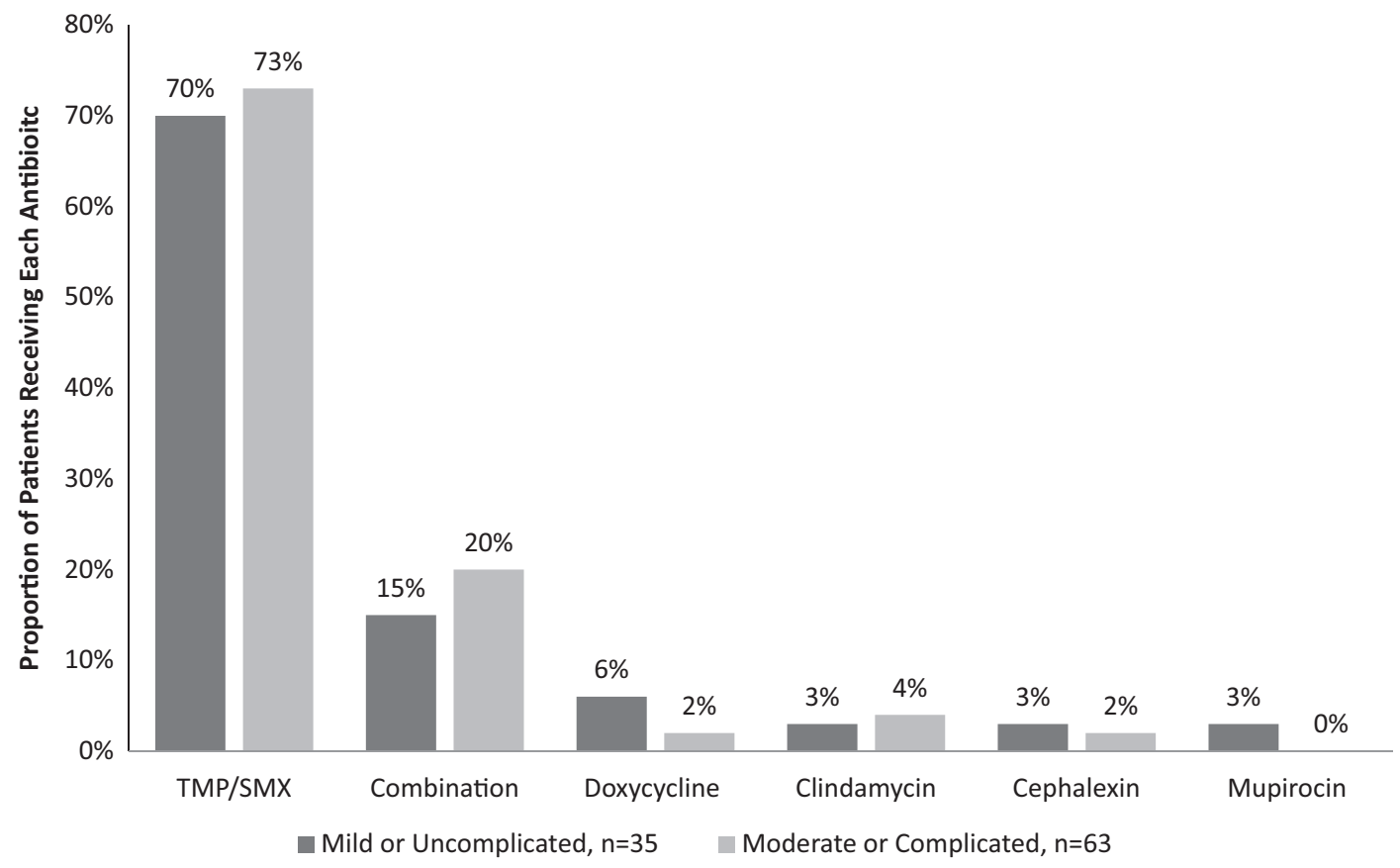

\footnotetext{
*There was no statistically significant difference in the initial antibiotic choice between patients with Mild or Uncomplicated infections as compared to patients with Moderate or Complicated infections (Chi-square test for homogeneity, $p=0.71$ ).

TMP/SMX, trimethoprim-sulfamethoxazole.
} 
Figure 4. Proportion of patients with treatment failure: patients receiving incision and drainage $(n=62) * *$

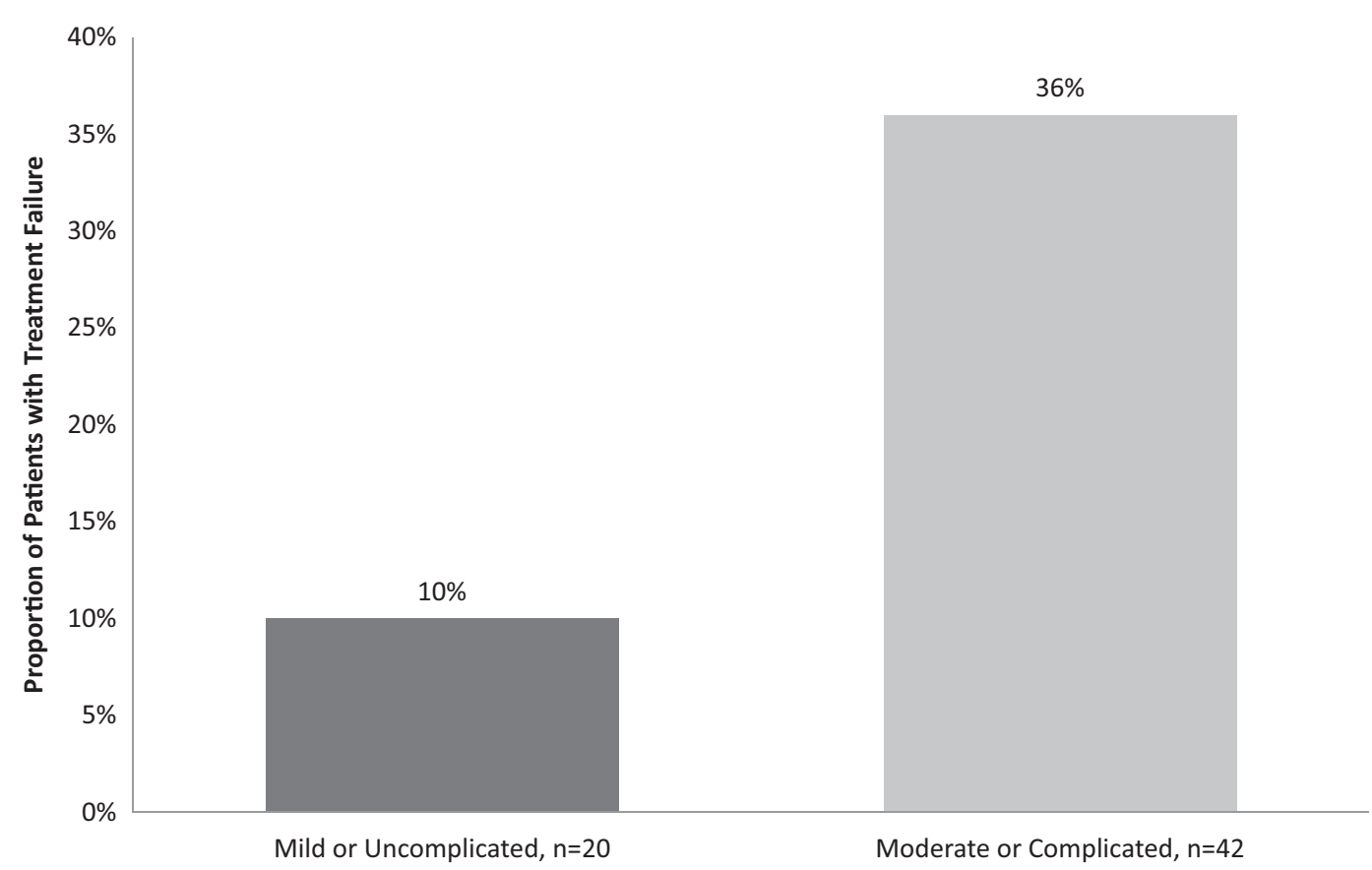

*There was a statistically significant difference in the rate of treatment failure between patients with Mild or Uncomplicated infections as compared to patients with Moderate or Complicated infections $(p=0.04)$.

days in the moderate or complicated severity group and 49.0 days in the mild or uncomplicated group $(P=.17)$. The mean additional cost associated with treatment failure was $\$ 2,174.05$ overall and was similar between the 2 severity groups (\$2,281.36 vs $\$ 2,132.46)$.

\section{Discussion}

This study measures the incidence of treatment failure and additional costs associated with failure for outpatients receiving oral antibiotics for MRSA SSTIs. This study reveals a high rate of treatment failure $(21 \%)$ and considerable associated costs (mean, \$1,933.71 per patient). These results suggest that baseline risk stratification and new treatment approaches are needed to reduce treatment failures and costs in the primary care setting.

\section{Incidence of Treatment Failure}

A previous study of $S$. aureus SSTIs treated in the outpatient setting demonstrated a treatment failure rate of $18 \%$, similar to that identified by our study. ${ }^{22}$ Other studies of MRSA SSTIs demonstrated higher rates of treatment failure.

A study by Menzin et $\mathrm{al}^{23}$ identified a treatment failure rate of $37 \%$; however, the study was conducted exclusively using hospitalized patients, who are likely to have more severe infections than outpatients. Furthermore, hospitalized patients are followed more closely by health care providers who are better able to detect complications and new infections. Indeed, studies have shown that outpatients may not be as likely as health care professionals to recognize new or recurrent skin infections, even with verbal and printed instructions. ${ }^{24,25}$ In addition, the treatment failure definition used by Menzin et al may have inflated the true rate of treatment failure. Menzin et al's definition classified patients who switched from intravenous to oral therapy as treatment failures. Transitioning from intravenous to oral antimicrobials is a common practice in hospitals and has been shown to have beneficial effects, including decreased costs, reduced incidence of catheter-related infections, and decreased hospital length of stay ${ }^{26-29}$; therefore, we do not believe this type of change should be considered to be a treatment failure.

A second study by Frei et $\mathrm{al}^{5}$ identified a treatment failure rate of $35 \%$; however, nearly half of the patients in that study were hospitalized. As previously mentioned, this might have resulted in greater identification of treatment failure compared with an outpatient population. In addition, the definition of treatment failure used by Frei et al in- 
cluded subsequent positive MRSA cultures as one of the criteria for treatment failure. This criterion was not used in the present study. When this additional criterion was eliminated, the failure rate reported by Frei et al becomes similar to that observed in the present study ( $25 \%$ vs $21 \%$ ).

\section{Additional Cost of Treatment Failure}

The patients in this study who failed therapy incurred additional health care costs of almost \$2,000 per patient. This is the first study to quantify this cost in the primary care setting. The mean cost of treatment failure observed in this cohort is considerably less than those costs described in prior cohorts. Other studies have calculated the cost of failure for hospitalized patients ${ }^{23}$ and patients managed at skilled nursing facilities or outpatient infusion clinics. ${ }^{22}$ Hospital room and board and outpatient infusion services accounted for 50\% and 25\% of the total costs in prior studies, respectively. Oral therapy may be another reason for the lower costs observed in this cohort. Studies by Menzin et $\mathrm{al}^{23}$ and Marton et $\mathrm{al}^{22}$ both demonstrated decreases in total cost of $12 \%$ and $30 \%$, respectively, when oral antibiotic therapy was chosen instead of intravenous antibiotic therapy.

This study also demonstrated higher treatment failure costs in patients with more severe MRSA SSTIs. This underscores the need for appropriate risk stratification at baseline.

\section{Validation of the Disease Severity Rule}

This study demonstrates that objective criteria available at baseline can be used to predict which patients are more likely to fail therapy with oral antibiotics. Simply put, patients with diabetes or lesions $\geq 5 \mathrm{~cm}$ in diameter were categorized as having moderate or complicated infections. $\mathrm{Pa}-$ tients with moderate or complicated infections had a failure rate more than twice as high as those patients with mild or uncomplicated infections. The difference was even more pronounced in a subgroup of patients who received I\&D. Two prior studies have reported higher rates of treatment failure in patients with lesions $\geq 5 \mathrm{~cm}$ in diameter. ${ }^{30,31}$ Furthermore, patients with diabetes have been shown to have more SSTI-related hospital and emergency department visits, longer hospital stays, and more infection-related deaths compared with nondiabetics. ${ }^{32,33}$ This simple rule provides primary care clinicians with a quick and easy clas- sification tool to estimate the risk of treatment failure in patients with an MRSA SSTI.

\section{Limitations}

This study has limitations. First, the cohort encompassed only patients in south Texas, which may limit the potential to generalize these results to other geographic regions. Second, there were more obese and Hispanic patients in the moderate or complicated severity group, likely reflecting a strong correlation between obesity, Hispanic ethnicity, and diabetes. Third, SSTIs can be caused by several pathogens, including staphylococci (MRSA and methicillin-sensitive $S$. aureus), streptococci, and Gram-negative bacteria. This study focuses on the subset of patients with cultures positive for MRSA. These patients may be more severely ill than the general population with SSTIs; therefore, the failure rate reported in this study of patients with MRSA SSTIs cannot be extrapolated to all patients with SSTIs. Fourth, 90-day follow-up information was captured retrospectively by the clinic personnel using the medical records available at their clinic. Patients who did not have additional visits (ie, did not return to the clinic or went to another clinic) were counted as treatment successes. In addition, clinicians may have changed their behavior to fit expected results of this observational study; a Hawthorne effect cannot be excluded. For these reasons, our study could have possibly underestimated the true rate of treatment failure. Finally, I\&D techniques were not standardized among the clinics or treating clinicians, and differences in I\&D techniques may have influenced SSTI outcomes.

Despite these limitations, this study has important strengths. It is the first to estimate the additional cost of MRSA SSTI treatment failure in the primary care setting. One in 5 patients presenting to a primary care clinic for the treatment of a MRSA SSTI will likely require additional intervention at an associated cost of almost $\$ 2,000$ per patient. In addition, objective criteria, available at baseline, can be used to predict which patients are more likely to need additional interventions. Ultimately, baseline risk stratification and new treatment approaches are needed to reduce treatment failures and costs in the primary care setting.

The authors thank their South Texas Ambulatory Research Network (STARNet) and Area Health Education Center col- 
leagues who assisted with the administrative aspects of the study: Holly Hayes, Stephanie Reyes, Marisa Rodriquez, and Paula Winkler. The authors also thank Kyllie Ryan-Hummel and Kyle Fischer (research assistants at the University of Texas Health Science Center, San Antonio), who formatted the final manuscript for submission to the fournal.

\section{References}

1. Klevens RM, Morrison MA, Nadle J, et al. Invasive methicillin-resistant Staphylococcus aureus infections in the United States. JAMA 2007;298:1763-71.

2. Klevens RM, Edwards JR, Tenover FC, McDonald LC, Horan T, Gaynes R. Changes in the epidemiology of methicillin-resistant Staphylococcus aureus in intensive care units in US hospitals, 1992-2003. Clin Infect Dis 2006;42:389-91.

3. Styers D, Sheehan DJ, Hogan P, Sahm DF. Laboratory-based surveillance of current antimicrobial resistance patterns and trends among Staphylococcus aureus: 2005 status in the United States. Ann Clin Microbiol Antimicrob 2006;5:2.

4. Moran GJ, Krishnadasan A, Gorwitz RJ, et al. Methicillin-resistant $S$. aureus infections among patients in the emergency department. N Engl J Med 2006; 355:666-74.

5. Frei CR, Miller ML, Lewis JS 2nd, et al. Trimethoprim-sulfamethoxazole or clindamycin for community-associated MRSA (CA-MRSA) skin infections. J Am Board Fam Med 2010;23:714-9.

6. Parchman ML, Munoz A. Risk factors for methicillin-resistant Staphylococcal aureus skin and soft tissue infections presenting in primary care: a South Texas Ambulatory Research Network (STARNet) study. J Am Board Fam Med 2009;22: 375-9.

7. Stevens DL, Bisno AL, Chambers HF, et al. Practice guidelines for the diagnosis and management of skin and soft-tissue infections. Clin Infect Dis 2005;41: 1373-406.

8. Liu C, Bayer A, Cosgrove SE, et al. Clinical practice guidelines by the Infectious Diseases Society of America for the treatment of methicillin-resistant Stapbylococcus aureus infections in adults and children. Clin Infect Dis 2011;52:e18-55.

9. Fridkin SK, Hageman JC, Morrison M, et al. Methicillin-resistant Staphylococcus aureus disease in three communities. N Engl J Med 2005;352: 1436-44.

10. Forcade NA, Wiederhold NP, Ryan L, Talbert RL, Frei CR. Antibacterials as adjuncts to incision and drainage for adults with purulent methicillin-resistant Staphylococcus aureus (MRSA) skin infections. Drugs 2012;72:339-51.

11. Eron LJ, Lipsky BA, Low DE, Nathwani D, Tice $\mathrm{AD}$, Volturo GA; Expert panel on managing skin and soft tissue infections. Managing skin and soft tissue infections: expert panel recommendations on key decision points. J Antimicrob Chemother 2003; 52(Suppl 1):i3-17.

12. US Department of Health and Human Services, Food and Drug Administration, Center for Drug Evaluation and Research (CDER). Guidance for industry: uncomplicated and complicated skin and skin structure infections-developing antimicrobial drugs for treatment. July 1998. Available from: http://www.fda.gov/ohrms/dockets/ac/03/ briefing/3997B1_02_GFI-Diabetic\%20Foot.pdf. Accessed February 9, 2012.

13. US Department of Health and Human Services, Food and Drug Administration, Center for Drug Evaluation and Research (CDER). Guidance for industry: acute bacterial skin and skin structure infections-developing antimicrobial drugs for treatment. Draft guidance. August 2010. Available from: http://www.fda.gov/downloads/Drugs/Guidance ComplianceRegulatoryInformation/Guidances/ UCM071185.pdf. Accessed February 9, 2012.

14. Corey GR, Stryjewski ME. New rules for clinical trials of patients with acute bacterial skin and skinstructure infections: do not let the perfect be the enemy of the good. Clin Infect Dis 2011;52(Suppl 7):S469-76.

15. Levy BT, Daly J. Community-acquired skin infections in the age of methicillin-resistant organisms. AHRQ Publication No. 11-0007-1-EF. Rockville, MD: Agency for Healthcare Research and Quality; 2010.

16. Forcade NA, Parchman ML, Jorgensen JH, et al. Prevalence, severity, and treatment of communityacquired methicillin-resistant Staphylococcus aureus (CA-MRSA) skin and soft tissue infections in 10 medical clinics in Texas: a South Texas Ambulatory Research Network (STARNet) study. J Am Board Fam Med 2011;24:543-50.

17. Merrill CT, Elixhauser A. HCUP fact book no. 6. Hospitalization in the United States, 2002. AHRQ publication no. 05-0056. June 2005. Available from: http://archive.ahrq.gov/data/hcup/factbk6/factbk6. pdf. Accessed March 5, 2010.

18. Machlin SR. Medicare Expenditure Panel Survey (MEPS). Statistical brief \#111. Expenses for a hospital emergency room visit, 2003. January 2006. Available from: www.meps.ahrq.gov/mepsweb/data files/publications/st111/stat111.pdf. Accessed March 5, 2010.

19. Machlin SR, Carper K. Medicare Expenditure Panel Survey (MEPS). Statistical brief \#166. Expenses for office-based physician visits by specialty, 2004. March 2007. Available from: www.meps.ahrq.gov/ mepsweb/data_files/publications/st166/stat166.pdf. Accessed March 5, 2010.

20. Russo CA, Owens P, Steiner C, Josephsen J. HCUP fact book no. 9. Ambulatory surgery in U.S. hospitals, 2003. AHRQ publication no. 07-0007. January 
2007. Available from: http://archive.ahrq.gov/data/ hcup/factbk9/factbk9.pdf. Accessed March 5, 2010.

21. US Department of Labor, Bureau of Labor Statistics. Archived Consumer Price Index detailed report information. Available from: www.bls.gov/cpi/ cpi_dr.htm. Accessed November 16, 2011.

22. Marton JP, Jackel JL, Carson RT, Rothermel CD, Friedman M, Menzin J. Costs of skin and skin structure infections due to Staphylococcus aureus: an analysis of managed-care claims. Curr Med Res Opin 2008;24:2821-8.

23. Menzin J, Marton JP, Meyers JL, Carson RT, Rothermel CD, Friedman M. Inpatient treatment patterns, outcomes, and costs of skin and skin structure infections because of Staphylococcus aureus. Am J Infect Control 2009;38:44-9.

24. Seaman M, Lammers R. Inability of patients to selfdiagnose wound infections. J Emerg Med 1991;9: 215-9.

25. Whitby M, McLaws ML, Collopy B, et al. Postdischarge surveillance: can patients reliably diagnose surgical wound infections? J Hosp Infect 2002;52: $155-60$.

26. Davis SL, Delgado G Jr, McKinnon PS. Pharmacoeconomic considerations associated with the use of intravenous-to-oral moxifloxacin for community-acquired pneumonia. Clin Infect Dis 2005;41(Suppl 2):S136-43.

27. Kuti JL, Le TN, Nightingale CH, Nicolau DP, Quintiliani R. Pharmacoeconomics of a pharmacistmanaged program for automatically converting levofloxacin route from i.v. to oral. Am J Health Syst Pharm 2002;59:2209-15.
28. Ramirez JA, Bordon J. Early switch from intravenous to oral antibiotics in hospitalized patients with bacteremic community-acquired Streptococcus pneumoniae pneumonia. Arch Intern Med 2001; 161:848-50.

29. Mertz D, Koller M, Haller P, et al. Outcomes of early switching from intravenous to oral antibiotics on medical wards. J Antimicrob Chemother 2009;64: 188-99.

30. Lee MC, Rios AM, Aten MF, et al. Management and outcome of children with skin and soft tissue abscesses caused by community-acquired methicillinresistant Staphylococcus aureus. Pediatr Infect Dis J 2004;23:123-7.

31. Frazee BW, Lynn J, Charlebois ED, Lambert L, Lowery D, Perdreau-Remington F. High prevalence of methicillin-resistant Staphylococcus aureus in emergency department skin and soft tissue infections. Ann Emerg Med 2005;45:311-20.

32. Bader MS. Diabetic foot infection. Am Fam Physician 2008;78:71-9.

33. Pallin DJ, Egan DJ, Pelletier AJ, Espinola JA, Hooper DC, Camargo CA Jr. Increased US emergency department visits for skin and soft tissue infections, and changes in antibiotic choices, during the emergence of community-associated methicillinresistant Staphylococcus aureus. Ann Emerg Med 2008; 51:291-8.

34. Alabama Medicaid Agency Average Acquisition Cost (AAC) rate listing for generic drugs: rates effective as of November 9, 2011. Available from: http:// al.mslc.com/AACList.aspx. Accessed December 1, 2011. 